\title{
Selective suppression and excitation of solid-state NMR resonances based on quadrupole coupling constants
}

\author{
Hyung-Tae Kwak, Subramanian Prasad, Ted Clark, and Philip J. Grandinetti* \\ Department of Chemistry, The Ohio State University, $120 \mathrm{~W}$. 18th Avenue, Columbus, OH 43210-1173, USA
}

Received 22 May 2002; revised 18 October 2002

\begin{abstract}
The dependence of the (Rotor Assisted Population Transfer) RAPT enhancement on offset frequency for nuclei experiencing different quadrupolar couplings has been exploited to design two new spectral editing schemes, $\pi / 2$-RAPT and RAPT- $\pi$-RAPT, for the selective excitation or suppression, respectively, of nuclei with large quadrupolar couplings. Both approaches are demonstrated on the ${ }^{87} \mathrm{Rb}$ spectrum of $\mathrm{Rb}_{2} \mathrm{SO}_{4}$, which contains two resonances with $C_{q}$ values of 2.6 and $5.3 \mathrm{MHz}$. The conditions for optimal selectivity are discussed. Combining $\pi / 2$-RAPT with the RIACT MQ-MAS experiment it is also demonstrated how a pure absorption mode triple quantum MQ-MAS spectrum devoid of narrow resonances can be obtained.
\end{abstract}

(C) 2003 Elsevier Science (USA). All rights reserved.

\section{Introduction}

While various approaches for spectral editing based on the magnitude of dipolar couplings have been developed in solid-state NMR, analogous methods based on the magnitude of quadrupolar couplings have not, until quite recently, appeared in the literature. In the specific case of MQ-MAS, Caldarelli and Ziarelli [1] recently showed that the strong offset dependence of multiple quantum to single quantum RIACT mixing can be used to selectively excite resonances with large quadrupolar couplings in the O-RIACT MQ-MAS experiment. This approach is quite useful, since signals associated with smaller quadrupolar couplings are the most easily excited and tend to dominate the MQ-MAS spectra. They also persist for longer duration in the MQ dimension resulting in longer experimental duration leading to signal truncations and related spectral distortions. For nuclei with weak quadrupolar couplings, $C_{q} \approx 0.5 \mathrm{MHz}$, accurate parameter determination may be obtained from single-pulse satellite transition MAS spectra of powders [2] and, thus, their resonances are undesirable in the MQ-MAS spectra. The limitations of O-RIACT include that it is a two-dimensional experi-

\footnotetext{
${ }^{*}$ Corresponding author. Fax: 614-292-1685.

E-mail address: grandinetti.1@osu.edu (P.J. Grandinetti).
}

ment requiring longer duration, and that structural features of the quadrupolar lineshapes are lost as only a fraction of the crystallites is excited.

In the case of static samples, Haase et al. [3] proposed a difference method where selective inversion of a satellite transition of a quadrupolar nucleus in a single crystal or oriented powder sample will selectively enhance its central transition. The difference spectrum between enhanced and unenhanced central transition spectra provides the spectrum of only the enhanced site. Limitations of this approach are that it requires a single crystal or oriented powder, and is not applicable under magic-angle spinning conditions.

We have recently developed a method called Rotor Assisted Population Transfer (RAPT) for enhancing MAS NMR sensitivity of quadrupolar nuclei in solids [4-6] by transferring polarization associated with satellite transitions to the central $m=1 / 2 \rightarrow-1 / 2$ transition. The advantage of the RAPT method over previous schemes [7-12] for utilizing satellite transition polarization is that RAPT can be applied to a polycrystalline sample during MAS to obtain simultaneous central transition enhancement of all crystallite orientations.

In this paper, we present a general approach, based on the RAPT enhancement scheme, to prepare an initial magnetization with sites selectively suppressed based on 
the size of their quadrupolar couplings. The advantages of this approach over that of Haase et al. [3] is that it can be applied to polycrystalline samples under MAS conditions. Additionally, the scheme prepares an initial magnetization with selective enhancement or suppression of sites, and does not require a difference experiment. Such a scheme can easily be incorporated into any experiment that draws its coherence from the central transition polarization. We also report results on a RAPT-RIACT scheme that has been modified to incorporate the RAPT-based schemes for selective suppression of resonances having small quadrupolar coupling constants.

\section{Experimental}

All NMR spectra were acquired at $9.4 \mathrm{~T}(130.939 \mathrm{MHz}$ ${ }^{87} \mathrm{Rb}$ frequency) with a Bruker DMX 400 spectrometer, using a Bruker $4 \mathrm{~mm}$ MAS probehead. The sample used for these polarization enhancement experiments was polycrystalline $\mathrm{Rb}_{2} \mathrm{SO}_{4}$, which has ${ }^{87} \mathrm{Rb}$ quadrupolar coupling parameters of $C_{q}=2.67 \mathrm{MHz}, \eta_{q}=0.89$, and $C_{q}=5.28 \mathrm{MHz}, \eta_{q}=0.13$ for $\mathrm{Rb}$ sites 1 and 2, respectively [13]. The solid-state ${ }^{87} \mathrm{Rb}$ resonance of $\mathrm{RbCl}$ was used to calibrate the radiofrequency field strength and as the external frequency reference $\left(\delta_{\mathrm{RbCl}}=0\right)$. All experiments were performed at spinning rates of $12 \mathrm{kHz}$.
The RAPT sequence for enhancing the central transition polarization, shown in Fig. 1a, consists of a train of Gaussian pulses $\left(\sigma=2.855 \mu \mathrm{s}, \tau_{\mathrm{p}}=13.5 \mu \mathrm{s}\right)$ with alternating off resonant frequencies of $\pm v_{\text {off }}$. This approach [6], which is a modification on the RAPT preparation described previously [4], overcomes the need for fast phase shifting and allows us to explore a wider range of frequency offsets. The use of Gaussian pulses also improves the selectivity of satellite excitation. A 2- $\mu$ s delay between each pulse in the RAPT pulse train was used to allow time for the transmitter phase to stabilize. The duration $\tau$ acts as a $z$ filter and is set to a small integer multiple of $T_{2}$ to dephase all coherences before the $\pi / 2$ pulse.

In Fig. $1 b$ is the $\pi / 2$-RAPT sequence for selective excitation of resonances with large quadrupolar couplings. To improve selectivity the first $\pi / 2$ pulse phase, $\phi_{1}$, is cycled in steps of $60^{\circ}$, with the receiver phase following the relationship:

$\phi_{\mathrm{R}}=0 \cdot \phi_{1}+\phi_{2}$.

In Fig. 1c is the RAPT- $\pi$-RAPT sequence for suppression of resonances with large quadrupolar couplings. To improve selectivity the $\pi$ pulse phase, $\phi_{1}$, is cycled in steps of $60^{\circ}$, with the receiver phase following the relationship in Eq. (1).

A bandpass filter was placed between the probe and the receiver to reduce the reflecting voltage from the

(a)

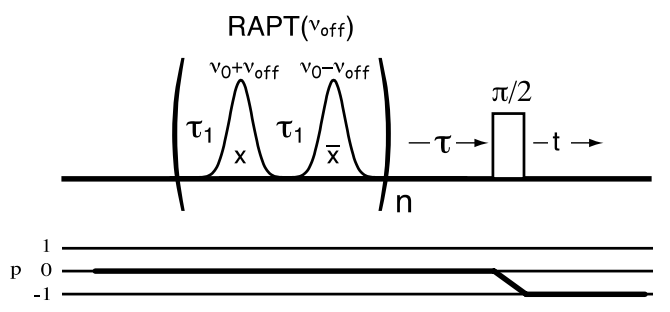

(b)
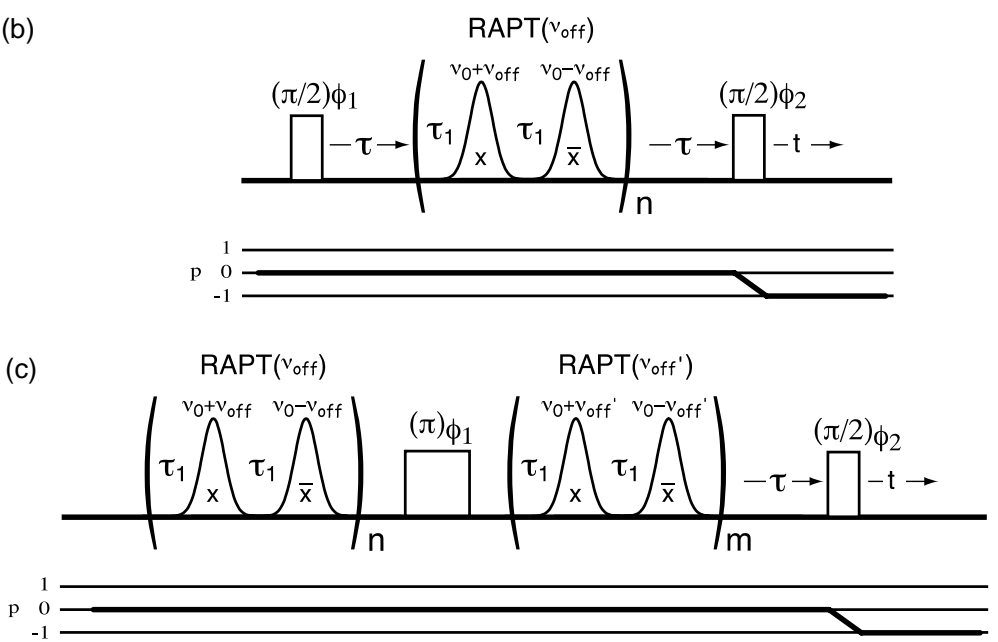

Fig. 1. (a) Rotor Assisted Population Transfer (RAPT) pulse sequence using off-resonance excitation and Gaussian-shaped pulses. (b) $\pi / 2-R A P T$ pulse sequence for suppression of resonances with small quadrupolar coupling constants. (c) RAPT- $\pi$-RAPT pulse sequence for suppression of resonances with large quadrupolar coupling constants. 


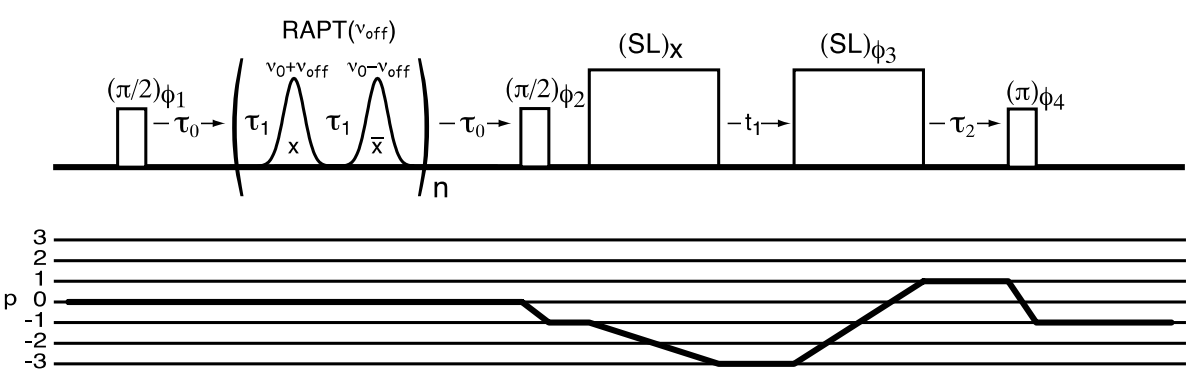

Fig. 2. $\pi / 2$-RAPT-RIACT(2) MQ-MAS pulse sequence and coherence transfer pathway for suppressing resonances with small quadrupolar coupling constants. For pathway selection see text for phase cycling details.

probe caused by the RAPT pulse train. An RF field strength of $73 \mathrm{kHz}$ was used for the RAPT pulse train, and the field strength for the presaturation pulse in the $\pi / 2$-RAPT experiments was $25 \mathrm{kHz}$.

The $\pi / 2$-RAPT sequence was employed in a shiftedecho [14] RIACT(2) experiment [15], as detailed in Fig. 2. To guarantee that the triple quantum coherence in the RIACT(2) is generated from the central transition polarization it was necessary to design a more robust phase cycling scheme. The simplest approach is to acquire signal from a single pathway as shown in Fig. 2. To select this pathway the pulse phases were varied independently in a 288 step phase cycle, each in steps of $\Delta \phi_{1}=90^{\circ}, \Delta \phi_{2}=60^{\circ}, \Delta \phi_{3}=30^{\circ}$, and $\Delta \phi_{4}=90^{\circ}$, with the receiver phase following the relation:

$\phi_{\mathrm{R}}=0 \cdot \phi_{1}+\phi_{2}-4 \cdot \phi_{3}+2 \cdot \phi_{4}$.

An RF field strength of $142 \mathrm{kHz}$ was used for the RAPT pulse train and the RIACT spin-locking pulses $\left(t_{r} / 4\right.$ duration). The initial $\pi / 2$ and final $\pi$ pulses on the central transition were performed using an RF field strength of $44 \mathrm{kHz}$. The RF field strength of the $\pi / 2$ pulse on the central transition prior to the RIACT spin lock pulse was $26 \mathrm{kHz}$. The RAPT offset frequency was set to $\pm 1390 \mathrm{kHz}$. All RIACT experiments were performed using a 500-ms recycle delay. The increment in the $t_{1}$ domain was rotor synchronized [16] to reduce experiment times. Since the offset dependence is large for sites having larger quadrupolar frequencies, the spinlocking field was set at the center of the lineshape arising from Rb-site 2.

\section{Results and discussion}

\subsection{Enhancement of signal intensities using RAPT preparation scheme.}

In previous work we have shown that the sensitivity of the NMR signal for the central transition of quadrupolar nuclei can be enhanced by transferring polarization from the satellite transitions by the selective saturation of these transitions using RAPT [4-6]. Fig. 3 shows a plot of the simulated RAPT sensitivity enhancement factor for the central transition of ${ }^{87} \mathrm{Rb}$ (spin $3 / 2$ ) as a function of $\left|v_{\text {off }}\right|$ for the two ${ }^{87} \mathrm{Rb}$ sites of $\mathrm{Rb}_{2} \mathrm{SO}_{4}$. For spin 3/2 nuclei, RAPT enhancements increase to a maximum near $\left|v_{\text {off }}\right| \approx\left|C_{q}\right| / 4$ and return to unity (no enhancement) when $\left|v_{\text {off }}\right| \geqslant\left|C_{q}\right| / 2$. With increasing $\eta_{q}$ the RAPT enhancement profile becomes broader but more important, it always returns to unity when $\left|v_{\text {off }}\right| \geqslant\left|C_{q}\right| / 2$, independent of $\eta_{q}$. This is a consequence of the outermost discontinuity (edge) of the first-order quadrupolar lineshape for a satellite transition depending only on $C_{q}$. Thus, measurement of the RAPT profile edge frequency, given by $v_{\text {edge }}=\left|3 C_{q}\right|$ $(2 I(2 I-1)) \mid$, where $I$ is the spin of the nucleus, provides a simple means for measuring the quadrupolar coupling constant [6]. In Fig. 3 an enhancement of 1.9 times for $\mathrm{Rb}$-site 1 is observed near the $C_{q} / 4$ value of $650 \mathrm{kHz}$, and an enhancement factor of 1.9 for Rb-site 2 is near the $C_{q} / 4$ value of $1300 \mathrm{kHz}$. The RAPT profile has a sharper gradient for the site 1 consistent with the site's larger $\eta_{q}$ value [6]. The vertical lines at 1300 and $2600 \mathrm{kHz}$ indicate the $v_{\text {edge }}$ values for each of the sites.

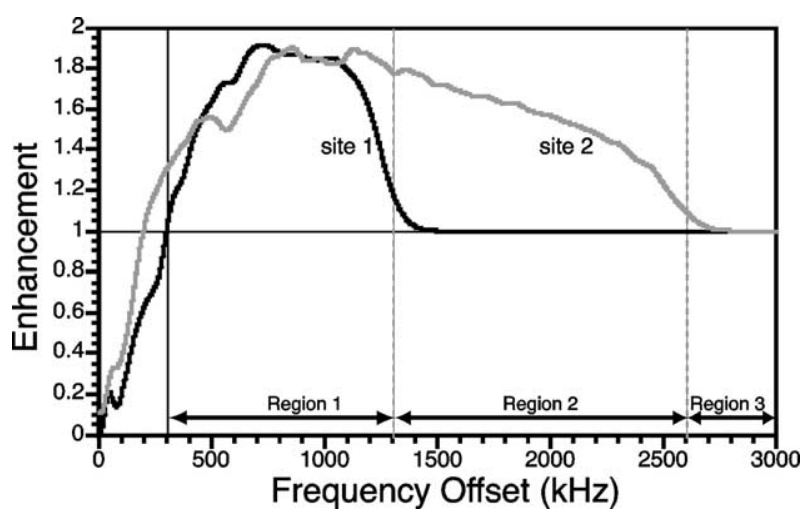

Fig. 3. Simulated Gaussian RAPT profiles for the ${ }^{87} \mathrm{Rb}$ sites in $\mathrm{Rb}_{2} \mathrm{SO}_{4}$. The RAPT preparation consisted of 4 Gaussian-shaped pulse pair at an RF field strength of $113 \mathrm{kHz}$. All simulations were based on a full numerical density matrix calculation and were averaged over 3722 crystallite orientations. The continuous motion of the rotor was approximated by discretizing each rotor period into 200 smaller time independent periods, according to standard methods [17]. 
Based on the differential offset dependence of the RAPT enhancement in this two site system we can divide the offset frequency into three regions, as indicated in Fig. 3. Referring to the smaller and larger sites as $C_{q}^{\mathrm{S}}$ and $C_{q}^{\mathrm{L}}$, respectively, clearly in the region 1 , where $\left|v_{\text {off }}\right| \ll\left|C_{q}^{\mathrm{S}} / 2\right|$ and $\left|v_{\text {off }}\right| \ll\left|C_{q}^{\mathrm{L}} / 2\right|$, both sites are enhanced. In region 2, where $\left|C_{q}^{\mathrm{S}} / 2\right| \ll\left|v_{\text {off }}\right| \ll\left|C_{q}^{\mathrm{L}} / 2\right|$, only the larger $C_{q}$ site is enhanced with the smaller $C_{q}$ site unaffected. In region 3 and beyond, when $\left|v_{\text {off }}\right| \gg\left|C_{q}^{\mathrm{S}} / 2\right|$ and $\left|v_{\text {off }}\right| \gg\left|C_{q}^{\mathrm{L}} / 2\right|$, neither site is enhanced.

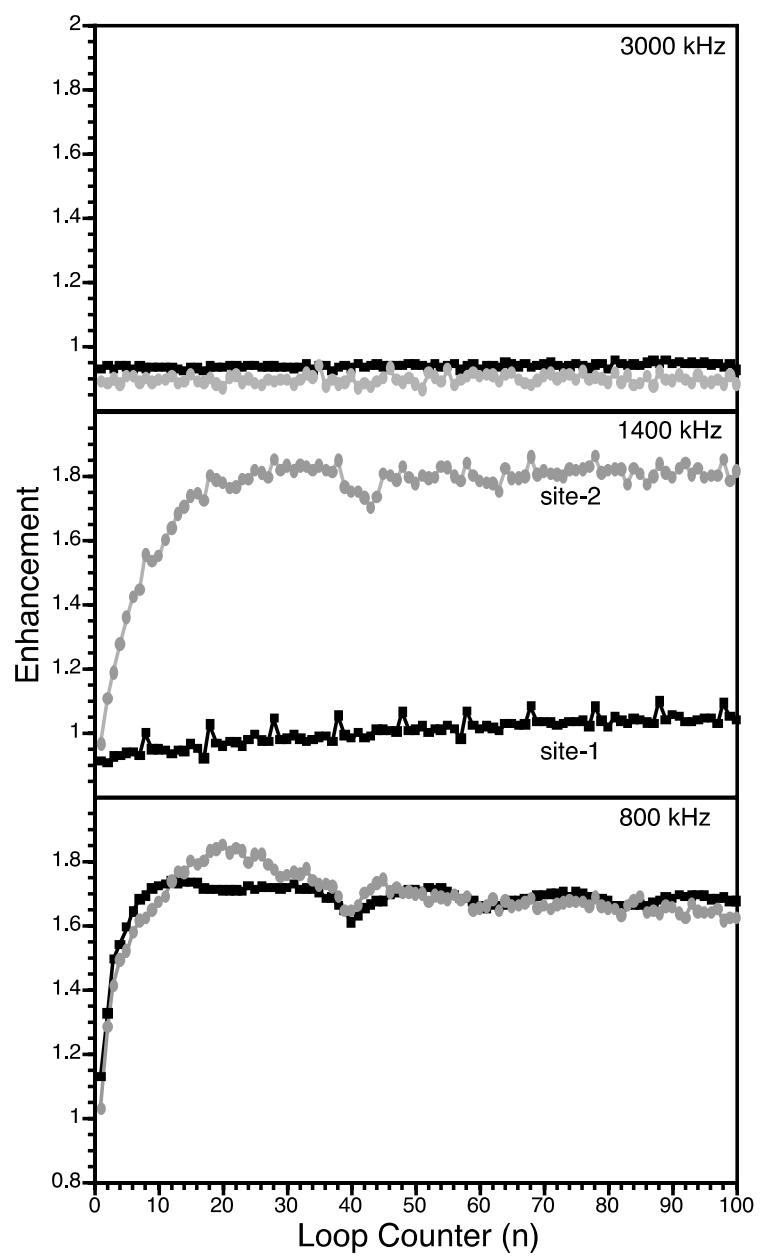

Fig. 4. RAPT enhancements as a function of loop counter for both $\mathrm{Rb}$ sites in $\mathrm{Rb}_{2} \mathrm{SO}_{4}$ at noted offset frequencies. The selective saturation of the satellite transitions for $\mathrm{Rb}$-site 2 is achieved in region 2 at $1400 \mathrm{kHz}$.
The experimental RAPT response as a function of loop counter, $n$, for both sites in all three regions at $\left|v_{\text {off }}\right|=800,1400$, and $3000 \mathrm{kHz}$ are shown in Fig. 4, and the results summarized in Table 1. At $\left|v_{\text {off }}\right|=800 \mathrm{kHz}$ (region 1), enhancement factors of 1.7 and 1.85 times are achieved for Rb-site 1 and $\mathrm{Rb}$-site 2 , respectively. At an offset frequency of $1400 \mathrm{kHz}$ (region 2 , beyond the $v_{\text {edge }}$ of $\mathrm{Rb}$-site 1) saturation of satellite populations for $\mathrm{Rb}$-site 2 occurs, avoiding the satellites of $\mathrm{Rb}$-site 1 . By increasing $n$ the RAPT enhancement factor increases to 1.85 . Beyond the $v_{\text {edge }}$ value of $\mathrm{Rb}$ site 2 (region 3), there is no enhancement as expected.

The varied responses to the RAPT preparation for the two $\mathrm{Rb}$ sites in $\mathrm{Rb}_{2} \mathrm{SO}_{4}$ based on their $C_{q}$ parameters, and the chosen offset frequency suggests that the $\mathrm{Rb}_{2} \mathrm{SO}_{4}$ spectrum may be manipulated by collecting spectra under different RAPT conditions. For example, one may compare a spectrum from region 2 with that of an unenhanced spectrum. Such spectra are shown in Fig. 5. The resonance with the smaller $C_{q}$ parameter was (largely) removed by taking the difference between these spectra, resulting in a spectrum of only the resonance of the larger $C_{q}$ value. Although this separation of sites is not quantitative, it does result in an isolated spectrum without significant distortions to the powder lineshape. With the current Gaussian RAPT excitation scheme we can selectively distinguish between sites whose $v_{\text {edge }}$ values differ by more than $300 \mathrm{kHz}$.

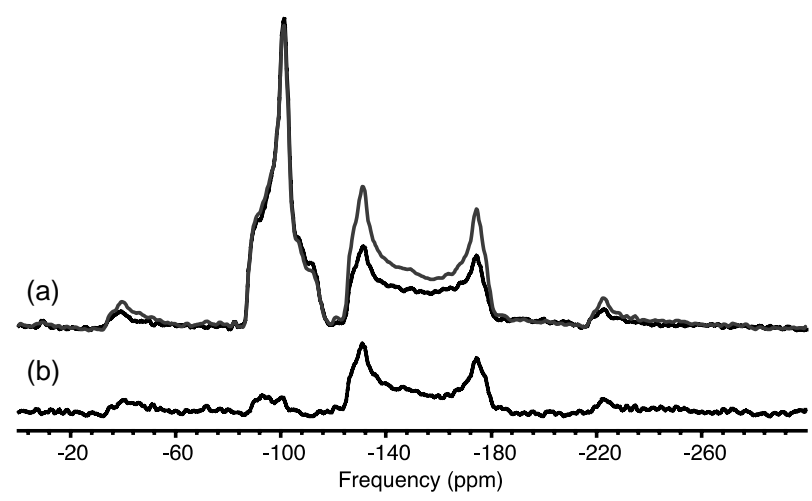

Fig. 5. In (a) the ${ }^{87} \mathrm{Rb}$ spectra of $\mathrm{Rb}_{2} \mathrm{SO}_{4}$ with and without selective RAPT enhancement of the large quadrupolar coupling resonance of $\mathrm{Rb}$-site 2. In (b) the difference spectrum allows one to isolate the ${ }^{87} \mathrm{Rb}$ spectrum of $\mathrm{Rb}$-site 2 .

Table 1

Experimental NMR parameters and RAPT parameters

\begin{tabular}{llllr}
\hline Description & Presaturation scheme & $v_{\text {off }}, v_{\text {off }}^{\prime}(\mathrm{kHz})$ & \multicolumn{2}{l}{ Enhancement } \\
\cline { 3 - 5 } & & & Rb-site 1 $\left(C_{q}=2.67\right)$ & $\mathrm{Rb}$-site 2 $\left(C_{q}=5.28\right)$ \\
\hline Enhancement of both sites & RAPT & 800 & 1.7 & 1.85 \\
Selective enhancement & RAPT & 1400 & 1.0 & 1.85 \\
Isolation of site 2 & $\pi / 2$-RAPT & 1400 & $\ll 0.1$ & 1.1 \\
Isolation of site 1 & RAPT- $\pi$-RAPT & 800,1400 & 1.0 & $\ll 0.1$ \\
\hline
\end{tabular}

The number of loops ( $n$ and $m$ in the pulse sequences, Fig. 1) were set to 27. Enhancements are given relative to a conventional Bloch decay experiment. 
While taking the difference of spectra with and without selective RAPT enhancement is a straightforward approach to suppress small $C_{q}$ resonances, we have developed more versatile approaches to prepare an initial central transition magnetization with the selective saturation of a central transition polarization based on the size of its quadrupolar coupling.

\subsection{Selective excitation or suppression of large quadru- polar couplings using RAPT.}

To prepare an initial central transition magnetization with the selective saturation of the resonances with small quadrupolar couplings we developed the $\pi / 2$-RAPT scheme outlined in Fig. 6. This approach initially presaturates the central transition of all sites by using a $\pi / 2$ pulse and then resurrects the magnetization of larger $C_{q}$ sites with a RAPT pulse train optimized (i.e., in region 2) for the large couplings. The pulse sequence implementing the $\pi / 2$-RAPT scheme is given in Fig. 1 b.

To prepare an initial central transition magnetization with the selective saturation of the resonances with large quadrupolar couplings we propose the RAPT- $\pi$-RAPT scheme outlined in Fig. 7. In this approach, two RAPT pulse trains separated by a $\pi$-pulse are employed. The first RAPT pulse train is optimized to enhance the central transition populations of all sites. This step is followed by a $\pi$ pulse that inverts the central transition

\section{$\pi / 2$-RAPT Scheme}

(Selective Excitation of Large Quadrupolar Couplings)

Small Coupling Populations

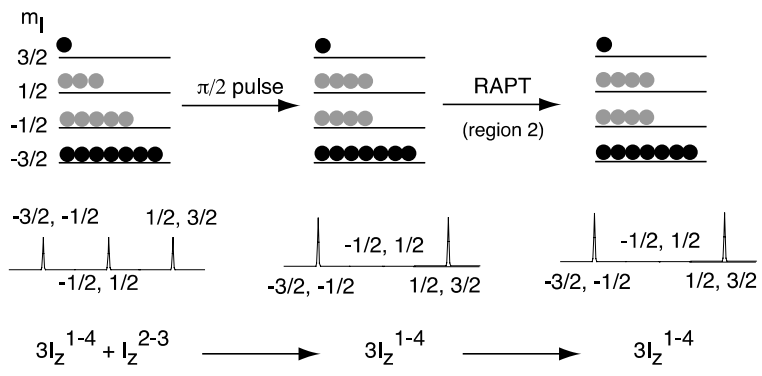

Large Coupling Populations

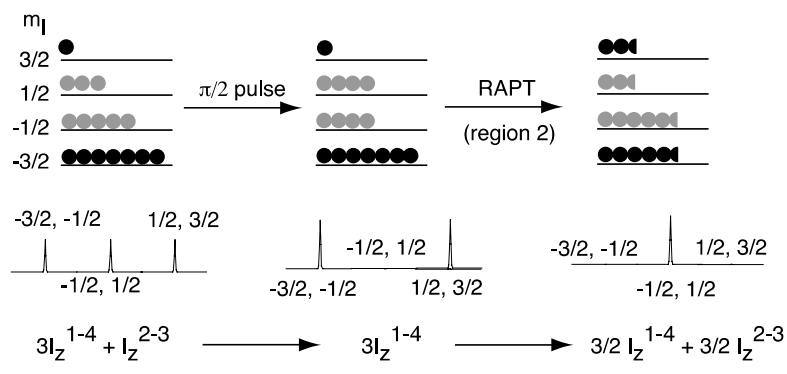

Fig. 6. Population manipulation during $\pi / 2$-RAPT.
RAPT- $\pi$ - RAPT Scheme

(Selective Suppresion of Large Quadrupolar Couplings)
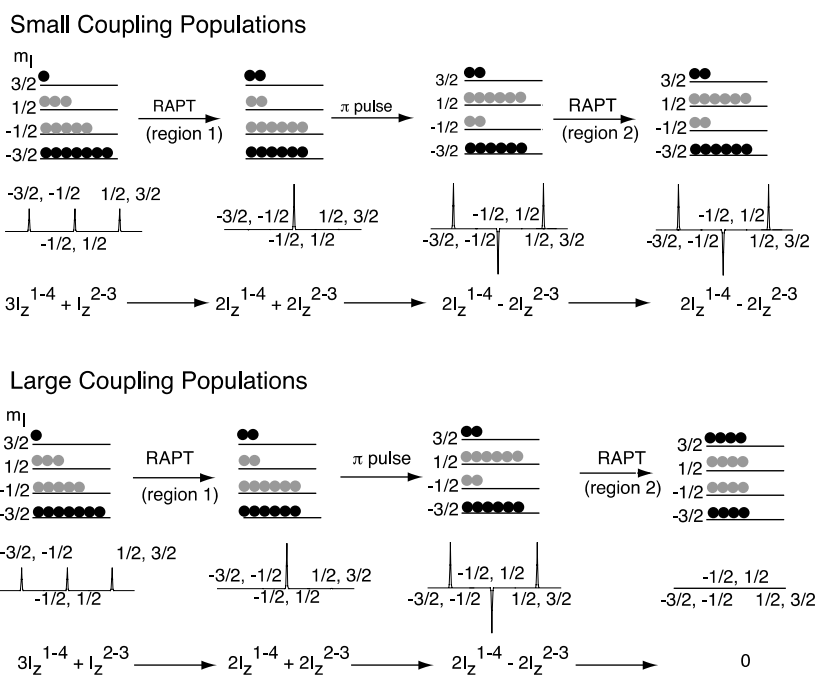

Fig. 7. Population manipulation during RAPT- $\pi$-RAPT.

populations of all sites. In the third step a selective RAPT pulse train, tuned to excite only the satellite transitions of sites with large quadrupolar couplings, is applied. The effect of this third and final step is to saturate the central transition of sites with large quadrupolar couplings, while leaving the enhanced magnetization in the central transition of sites with small quadrupolar couplings unaffected. The pulse sequence implementing the RAPT- $\pi$-RAPT scheme is given in Fig. 1c.

An example of spectra collected using both approaches on the two ${ }^{87} \mathrm{Rb}$ resonances in $\mathrm{Rb}_{2} \mathrm{SO}_{4}$ is shown in Fig. 8 along with the conventional Bloch decay

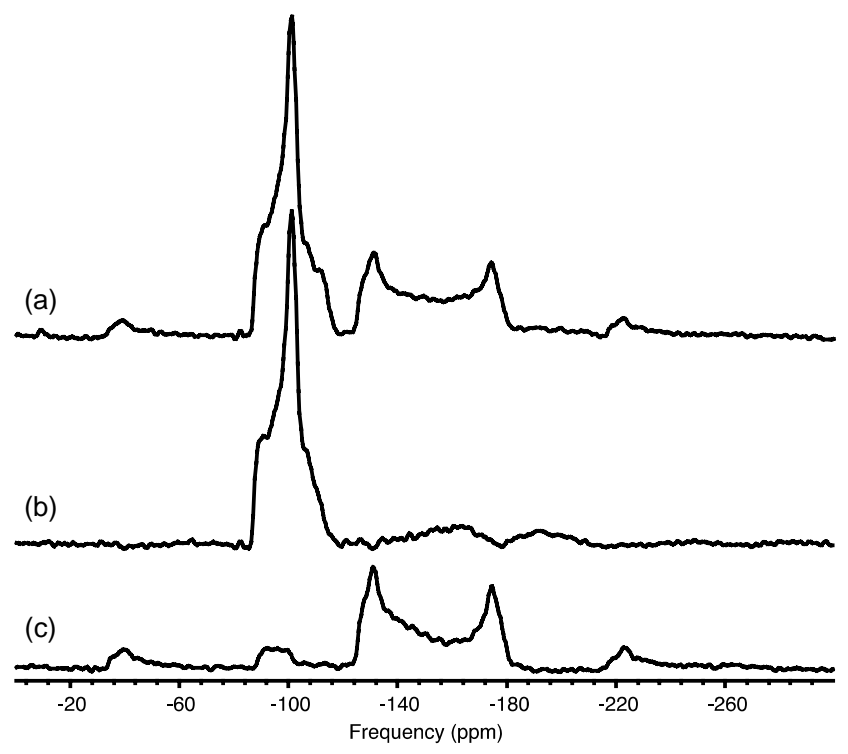

Fig. 8. (a) Conventional Bloch decay ${ }^{87} \mathrm{Rb}$ spectrum of $\mathrm{Rb}_{2} \mathrm{SO}_{4}$. (b) Selective excitation of the smaller quadrupolar coupling resonance using the RAPT- $\pi$-RAPT pulse sequence. (c) Selective excitation of the larger quadrupolar coupling resonance using the $\pi / 2$-RAPT pulse sequence. 
(a)
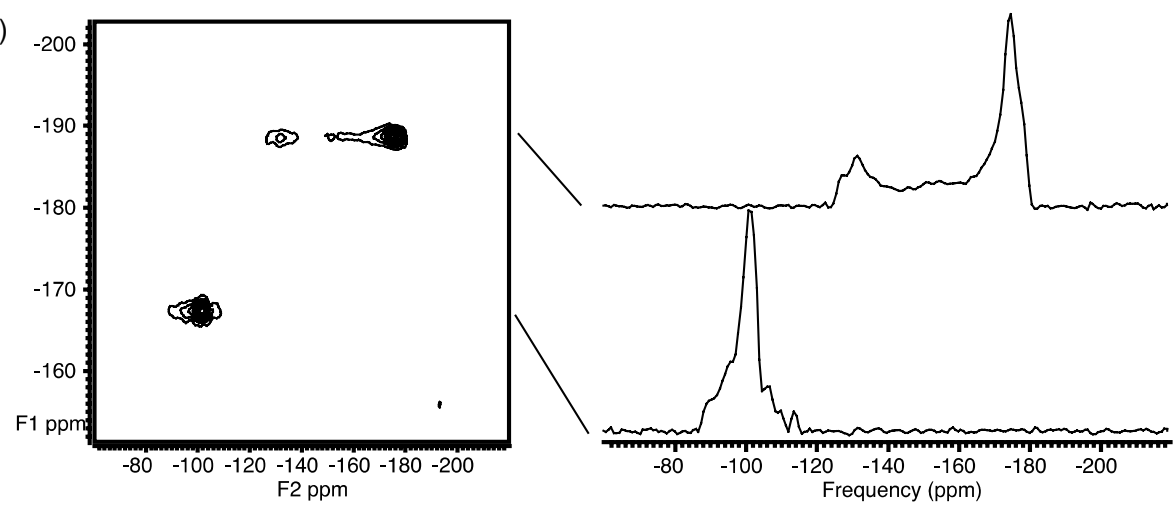

(b)
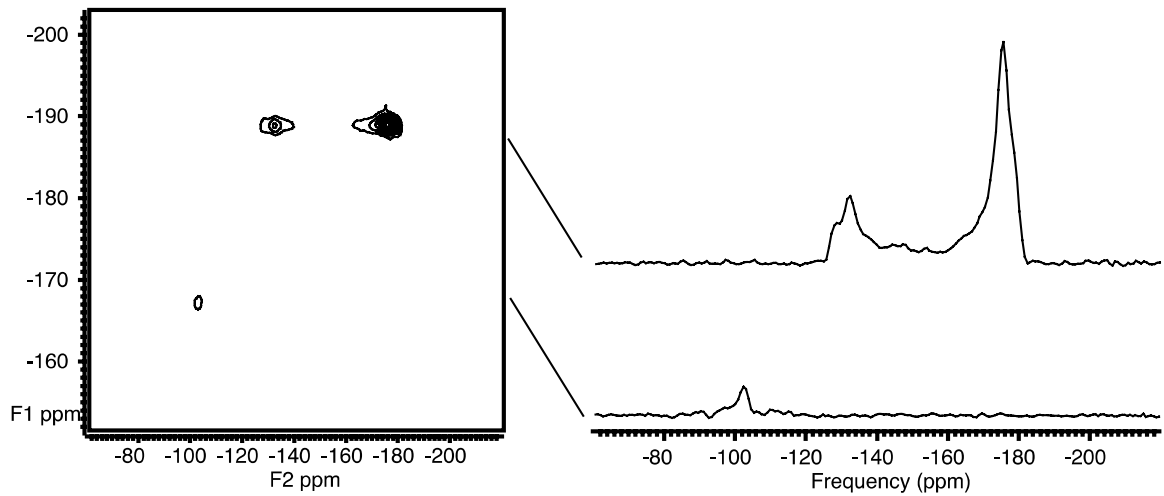

Fig. 9. Two-dimensional (a) RIACT and (b) $\pi / 2$-RAPT-RIACT spectra. The right-hand side shows the F2 projections of the respective sites. A total of 25 points with a dwell of $83.33 \mu$ s were acquired in the $t_{1}$ domain. A line broadening of $200 \mathrm{~Hz}$ was applied in the $t_{1}$ domain and zero filled twice. The 20 contour levels span from 0 to $100 \%$ of the maximum intensity.

spectrum. In practice, we found it is difficult to invert the central transition populations in the RAPT- $\pi$-RAPT experiment in a quantitative manner and so the final intensity of Rb-site 1 is less than that predicted by Fig. 7 (being approximately equal to the Bloch decay, instead of enhanced by a factor of 1.7). Nevertheless, this approach is quite effective in discriminating between sites with different $C_{q}$ values and in preserving the expected lineshape. In both experiments we obtain selective excitation of sites with small or large quadrupolar couplings, respectively, with minimal lineshape distortions.

\subsection{Site discrimination in RIACT experiments using $R A P T$ preparation scheme}

Since the RIACT(2) MQ-MAS experiment [15] draws its coherence from the central transition polarization it can be prefixed with RAPT [5]. Sensitivity enhancement factors as high as 1.8 have been reported $[5,18,19]$ for spin $3 / 2$ nuclei using such an approach.

As mentioned earlier, triple quantum spectra of samples containing narrow resonances hinder the acquisition of pure absorption mode spectra. The $\pi / 2-$ RAPT sequence is an ideal choice to rectify the problems associated with truncation and spectral distortions in the RIACT spectra by eliminating the narrow resonances. As a simple illustration, the 2D RIACT spectra of $\mathrm{Rb}_{2} \mathrm{SO}_{4}$ with and without $\pi / 2$ RAPT preparation, along with the projections in the F2 dimensions, are given Fig. 9. Both spectra are presented on the same vertical scale. Without $\pi / 2$ RAPT preparation, both the sites are present in the spectrum shown in Fig. 9a. In contrast, the $\pi / 2$-RAPT preparation allows us to scale down the intensity of the $\mathrm{Rb}$-site 1 to $10 \%$ while the intensity of the $\mathrm{Rb}$-site 2 is unaffected. Thus, the $\pi / 2$-RAPT-RIACT scheme reduces contributions from narrow signals and separates the broad signals in the isotropic dimension. The method also reduces the experimental time, and eliminates truncation of the $t_{1}$ domain signals that results in spectral distortions.

Of course, RAPT- $\pi$-RAPT could also be used to reduce contribution from broad resonances while retaining the narrow resonances in the RIACT experiments. We felt, however, that this approach would be of limited utility and choose not to present those results here.

\section{Conclusion}

The dependence of the RAPT enhancement on offset frequency for nuclei experiencing different quadrupolar 
couplings has been exploited to design two new spectral editing schemes, $\pi / 2$-RAPT and RAPT- $\pi$-RAPT, for the selective excitation or suppression, respectively, of nuclei with large quadrupolar couplings. We have demonstrated that both approaches can be used successfully in the ${ }^{87} \mathrm{Rb}$ spectrum of $\mathrm{Rb}_{2} \mathrm{SO}_{4}$, which contains two resonances with $C_{q}$ values of 2.6 and $5.3 \mathrm{MHz}$. Although not entirely quantitative, both approaches are advantageous in that they can isolate spectra of specific sites without significant anisotropic lineshape distortions or increases in experimental acquisition time. The schemes described in this investigation, in light of their ease of implementation, could be considered as an alternative approach for resolving overlapping spectral features in the NMR spectra of quadrupolar nuclei in polycrystalline solids. Generally, either technique can be applied to any solid-state NMR experiment that draws its coherence from the polarization of the central transition. Using $\pi / 2$-RAPT we have also demonstrated how the pure absorption mode triple quantum RIACT spectra devoid of signals associated with narrow resonances can be obtained.

\section{Acknowledgments}

This material is based upon work supported partly by the National Science Foundation under Grant No. CHE 0111109, the US Department of Energy, and the Ohio Supercomputer Center. Any opinions, findings and conclusions or recommendations expressed in this material are those of the author(s) and do not necessarily reflect the views of the National Science Foundation (NSF).

\section{References}

[1] S. Caldarelli, F. Ziarelli, Spectral editing of solid-state MAS NMR spectra of half-integer quadrupolar nuclei, J. Am. Chem. Soc. 122 (2000) 12015-12016.

[2] J. Skibsted, N.C. Nielsen, H. Bildsoe, H.J. Jakobsen, Satellite transitions in MAS NMR spectra of quadrupolar nuclei, J. Magn. Reson. 95 (1991) 88-117.

[3] J. Haase, N.J. Curro, R. Stern, C.P. Slichter, New methods for NMR of cuprate superconductors, Phys. Rev. Lett. 81 (1998) $1489-1492$.
[4] Z. Yao, H.-T. Kwak, D. Sakellariou, L. Emsley, P.J. Grandinetti, Sensitivity enhancement of the central transition NMR signal of quadrupolar nuclei under magic-angle spinning, Chem. Phys. Lett. 327 (2000) 85-90.

[5] H.-T. Kwak, S. Prasad, Z. Yao, P.J. Grandinetti, J.R. Sachleben, L. Emlsey, Enhanced sensitivity in RIACT/MQ-MAS NMR experiments using rotor assisted population transfer, J. Magn. Reson. 150 (2001) 71-80.

[6] S. Prasad, H.T. Kwak, T. Clark, P.J. Grandinetti, A simple technique for determining nuclear quadrupole coupling constants using RAPT solid-state NMR spectroscopy, J. Am. Chem. Soc. 124 (18) (2002) 4964-4965.

[7] R.V. Pound, Nuclear electric quadrupole interactions in crystals, Phys. Rev. 79 (1950) 685-702.

[8] S. Vega, Y. Naor, Triple quantum NMR on spin systems with $I=3 / 2$ in solids, J. Chem. Phys. 75 (1981) 75.

[9] J. Haase, M.S. Conradi, Sensitivity enhancement for NMR of the central transition of quadrupolar nuclei, Chem. Phys. Lett. 209 (3) (1993) 287-291.

[10] J. Haase, M.S. Conradi, E. Oldfield, Single- and double-resonance experiments of quadrupolar nuclei in solids using sensitivity enhancement of the central transition, J. Magn. Reson. A 109 (1994) 210-215.

[11] J. Haase, M.S. Conradi, C.P. Grey, A.J. Vega, Population transfers for NMR of quadrupolar spins in solids, J. Magn. Reson. A 109 (1994) 90-97.

[12] A.P.M. Kentgens, R. Verhagen, Advantages of double frequency sweeps in static, MAS, and MQMAS NMR of $\operatorname{spin} I=3 / 2$ nuclei, Chem. Phys. Lett. 300 (1999) 435-443.

[13] T. Vosegaard, J. Skibsted, H. Bildsoe, H.J. Jakobsen, Quadrupole coupling and anisotropic shielding from single-crystal NMR of the central transition for quadrupolar nuclei, ${ }^{87} \mathrm{Rb} \mathrm{NMR}$ of $\mathrm{RbClO}_{4}$ and $\mathrm{Rb}_{2} \mathrm{SO}_{4}$, J. Magn. Reson. 122 (1996) 111-119.

[14] P.J. Grandinetti, J.H. Baltisberger, A. Llor, Y.K. Lee, U. Werner, M.A. Eastman, A. Pines, Pure absorption-mode lineshapes and sensitivity in two-dimensional dyanmic angle spinning NMR, J. Magn. Reson. A 103 (1993) 72-81.

[15] G. Wu, D. Rovnyak, R.G. Griffin, Quantitative multiple-quantum magic-angle spinning NMR spectroscopy of quadrupolar nuclei in solids, J. Am. Chem. Soc. 118 (1996) 9326-9332.

[16] D. Massiot, Sensitivity and lineshape improvements of MQ-MAS by rotor-synchronized data acquisition, J. Magn. Reson. A 122 (1996) 240.

[17] P. Hodgkinson, L. Emsley, Numerical simulation of solid-state NMR experiments, Prog. Nucl. Magn. Reson. Spectrosc. 36 (3) (2000) 201-239.

[18] K.H. Lim, T. Charpentier, A. Pines, Efficient triple-quantum excitation in modified RIACT MQMAS NMR for $I=3 / 2$ nuclei, J. Magn. Reson. 154 (2002) 196-204.

[19] P.K. Madhu, M.H. Levitt, Signal enhancement in the triplequantum magic-angle spinning NMR of spins-3/2 in solids: The FAM-RIACT-FAM sequence, J. Magn. Reson. 155 (2002) 150155. 